\title{
There Can be a Syntax of Thought Related to Logical Forms
}

\author{
Miguel López-Astorga
}

Ph.D. in Logic and Philosophy of Science Institute of Humanistic Studies "Juan Ignacio Molina", University of Talca, Chile

Email: milopez@utalca.cl

\section{Doi:10.5901/mjss.2017.v8n1p270}

\begin{abstract}
Nowadays, a very important theory, the mental models theory, is demonstrating that it is able to explain most of the results in reasoning experiments reported by the cognitive science literature. However, this has a consequence. The mental models theory is mainly focused on content and meaning, and its theses can lead to reject the idea that syntax plays a role in the human mind and that reasoning is logical. But, in this paper, I try to show that it is possible to accept the basic framework of the mental models theory and, at the same time, to continue to claim that there are syntactic and formal logical processes coherent with the way our mind works. To do that, I argue that, even accepting that the mental models theory describes correctly the processes why certain combinations of possibilities are detected, it can be stated that the relationships between such combinations indicated by the theory are consistent with, for example, the modal axiomatic system $\mathrm{K}$.
\end{abstract}

Keywords: axiomatic system; meaning; mental models; modal logic; system $\mathrm{K}$

\section{Introduction}

The idea of a syntax of thought is very important in certain formal approaches about human reasoning, for example, the one of the mental logic theory (e.g., Braine \& O'Brien, 1998a; O'Brien, 2009, 2014; O'Brien \& Li, 2013), which, as far as this idea is concerned, is based in turn on frameworks such as those of Fodor (1975) and Macnamara (1986). In this way, especially relevant works can be, for instance, that of Braine and O'Brien (1998b, pp. 47ff) or that of O'Brien and Li (2013, pp. 27ff). Likewise, papers such as, for example, the one of López-Astorga (2016a, pp. $12 \mathrm{ff})$ also explain and develop this thesis. But what is relevant here is that this idea, which leads to think that human reasoning is about logical forms, is in crisis at present. Maybe the main motive of that is the success of other rival theory adopting semantic assumptions that can cause the need of a syntax in the human mind to be ignored. That theory is that of the mental models (e.g., Byrne \& Johnson-Laird, 2009; Johnson-Laird, 2006, 2010, 2012, 2015; Johnson-Laird, Khemlani, \& Goodwin, 2015; Khemlani, Orenes, Johnson-Laird, 2012, 2014; Oakhill \& Garnham, 1996; Orenes \& Johnson-Laird, 2012; Ragni, Sonntag, \& Johnson-Laird, 2016). Indeed, this theory appears to raise great difficulties to the syntactic approaches such as that of the mental logic, and this is so for at least one important reason. If we pay attention to the cognitive science literature, we can note that the mental models theory predicts many more experimental results than the syntactic frameworks, which suggests that it is very possible that the human reasoning activity has nothing to do with logical forms, since the mental models theory propose that the human mind works taking into account basically just combinations of semantic possibilities and comparing them.

However, in my view, it can be thought that, even if we assume the main theses of the mental models theory, it is possible to speak about a deep or underlying syntax in the human mind. The reason of this is that, while, as held by the mental models theory, the identification of combinations of possibilities can be a clearly semantic process, the relationships that, subsequently, can be provided between those combinations in order to draw conclusions refer to processes that can be considered to be syntactic. But what is truly surprising in this regard is that those relationships and processes are largely coherent with, for instance, the general framework of the modal axiomatic system $\mathrm{K}$, which in turn means that they may also not be inconsistent with logical calculi such as that of natural deduction presented by Gentzen (1935), since, as it is known, K assumes standard logic.

To show this is the goal of this paper. To achieve it, I will firstly describe the general theses of the mental models theory and its proponents' arguments to hold that the comparison of semantic possibilities is enough to explain human reasoning. Secondly, I will account for how the theory exactly works in practice by means of some examples of inferences. Then I will also try to indicate how, from the combinations of possibilities identified by the theory, formulae in the language of $\mathrm{K}$ can be built. Finally, I will demonstrate that this last system can leads to conclusions akin to those 
predicted initially by the mental models theory. This last point will be explained with the help of the same examples used previously.

\section{The Mental Models Theory and the Combinations of Possibilities}

True, the mental models theory is currently a very relevant approach that is able to explain many phenomena that other approaches cannot (see, e.g., Orenes \& Johnson-Laird, 2012). In fact, it has been used even to reinterpret ancient logical theories or clarify some of its points (see, e.g., López-Astorga, 2015a, 2016b, 2016c). For this reason, my aim here is not to challenge the theory, since its potential and its possibilities are obvious. As indicated, my intention is only to show that, although its theses on content and meaning are accepted, that does not necessarily lead us to reject the idea of a syntax of thought. But, evidently, to do that, it is essential firstly to comment on which those theses are. The truth is that they are to be found in several papers, books, and chapters of books. In this way, although, for instance, Johnson-Laird's (2012) chapter is only one of those works, it can be illustrative enough, as it includes a very interesting table (Table 9.2) that indicates the combinations of possibilities corresponding to each connective in standard logic (conjunction, disjunction, conditional,...).

However, before describing such combinations, it is necessary to highlight a basic differentiation that the theory provides. That is the differentiation between 'Mental Models' and 'Fully Explicit Models'. A Mental Model is "an iconic representation of a possibility that depicts only those clauses in a compound assertion that are true" (Johnson-Laird et al., 2015, p. 202). On the other hand, a Fully Explicit Model "...represents a possibility depicting each clause in the premises as either true or not" (Johnson-Laird et al., 2015, p. 202). It is hence obvious that the Fully Explicit Models are harder to detect, since they give more information, and that individuals not always identify them. They often detect only the Mental Models, as that is much easier.

In this way, coming back to Johnson-Laird (2012, p. 138) and his Table 9.2, it can be said that, in the case of a conjunction such as ' $p$ and $q$ ', it is only possible to speak about one Mental Model, which fits its only Fully Explicit Model too. That is as follows:

$\mathrm{p}$

q

This model, evidently, stands for a situation in which both $p$ and $q$ are true, that is, the only situation in which a conjunction can be true. But the case of an exclusive disjunction is different. Given that the Mental Models, as mentioned, only refer to what is true, the Mental Models of an exclusive disjunction such as ' $p$ or $q$ and not both of them' are:

$\mathrm{p}$

The first model represents the situation in which $p$ is true and the second one corresponds to the scenario in which $q$ is so. However, the Fully Explicit Models complete the information:

$\mathrm{p}$

$\neg p$ $\neg q$

q

Where ' 7 ' stands for negation.

Now, what is false is also represented, that is, the first model not only shows that $p$ is true, but also that $q$ is false. Likewise, the second model informs both that $q$ is true and that $p$ is false.

It is clear, on the other hand, that if the disjunction were inclusive, that is, if it were ' $p$ or $q$ or both of them', it would have to include, in addition, a model such as that of conjunction (i.e., a model in which both $p$ and $q$ were true) both in the Mental Models set and in the Fully Explicit Models set. This is so because in that model neither $p$ nor $q$ are denied. So, it can belong both to the Mental Models and to the Fully Explicit Models of the inclusive disjunction.

Thus, the case of the conditional is easy to understand. Its Mental Models set include, again, only the case in which the clauses are true:

$\mathrm{p}$

But the Fully Explicit Models also refer to other two situations in which, despite the fact that there are negations, the conditional in entirety can be true: 
$\begin{array}{ll}\neg p & q \\ \neg p & \neg q\end{array}$

Table 9.2 in Johnson-Laird (2012, p. 138), which does not use the letters ' $p$ ' and ' $q$ ', but 'A' and 'B', also describes other connectives, but those indicated can be enough for the goal of this paper. Perhaps it is only necessary to add now that another important concept of the theory is that of modulation. This concept refers to the fact that, although combinations such as those mentioned are the habitual combinations for the connectives explained, people do not always assign those combinations to those connectives, since "...content, context, or knowledge can prevent the construction of a model and can add information to a model" (Johnson-Laird et al., 2015, p. 202). This can be clearly seen in the following example:

"If she played a musical instrument then she didn't play a flute" (Johnson-Laird, 2010, p. 201).

In principle, one might think that, because it is a conditional, this sentence should have one Mental Model:

\section{Musical instrument}

And three Fully Explicit Models:

Musical instrument

$\neg$ (Musical instrument)

$\neg$ (Musical instrument) $\neg$ (Flute)

$\neg$ (Flute)

$\neg$ (Flute)

Flute

Nonetheless, the last Fully Explicit Model is impossible, as it is not possible to play a flute and not to play a musical instrument. So, modulation removes this last model and, finally, the sentence only has two Fully Explicit Models (the way the mental models theory considers this example is also explained, e.g., in López-Astorga, 2016b, p. 18).

Of course, more aspects of the theory could also be dealt with here. For example, the way it considers the negated sentences (e.g., Khemlani et al., 2012, 2014). However, the points described enable to develop my explanation without the need to make this paper excessively extensive. In this way, what is interesting now is to see how the theory works in practice.

\section{The inferential activity following the mental models theory}

The best way to see how the mental models theory explains the human inferential activity is by means of examples. Let us think about the following inference:

"If there is a triangle then there isn't a circle.

There is a circle

So, there isn't a triangle" (Johnson-Laird, 2010, p. 201).

According to the theory, to make this kind of inference (which, as it is known, is an instance of Modus Tollendo Tollens) has an especial difficulty, since, to do that, it is necessary to identify the Fully Explicit Models of the first premise and its Mental Model is not enough (see, e.g., Byrne \& Johnson-Laird, 2009, pp. 282-283). Indeed, the Mental Model only gives this possible situation:

Triangle

$\neg$ (Circle)

And the second premise proposes a scenario in which there is a circle. So, individuals need to make effort and display all of the Fully Explicit Models:
Triangle
$\neg$ (Triangle)
$\neg($ Triangle)
$\neg$ (Circle)
$\neg($ Circle)
Circle

Thus, people can note that, in the only scenario in which it is true that, as provided by the second premise, there is 
a circle (the third Fully Explicit Model), it is not possible that there is a triangle. Therefore, the expected conclusion is, if all of the Fully Explicit Models are detected, that, certainly, there is not a triangle.

But the sentence used as an example in the previous section can also be useful here:

"If she played a musical instrument then she didn't play a flute.

She played a flute.

So, she didn't play a musical instrument" (Johnson-Laird, 2010, p. 201).

As it can be noted, this inference has the same formal structure as the previous one (it matches Modus Tollendo Tollens again). Nevertheless, according to the theory, it is not probable that individuals come to the conclusion in this case. The reason is that commented on above. The Fully Explicit Models of the first premise are only two, and the one necessary to derive the conclusion, the third one, the one in which she did not play a musical instrument and she did play a flute, is impossible. This is one of the strengths of the theory and, according to its proponents, a clear advantage over the syntactic or formal frameworks. True, a theory claiming that people reasoning following, for example, Gentzen's (1935) calculus could not account for this fact. In that calculus, these two last inferences have exactly the same structure, which is this one:

$$
\begin{aligned}
& (p \rightarrow \neg q) \\
& q \\
& \neg p
\end{aligned}
$$

And, as it is well known, in standard logic, [p $\rightarrow \neg q, q] \vdash[\neg p]$, no matter the contents of [p] and [q] (as said, a similar account in this regard is to be found, e.g., in López-Astorga, 2016b, p. 18, too).

Undoubtedly, the mental models theory seems to be right here, since it is hard to suppose that somebody will agree with the conclusion in the second example, and this is a real problem that the syntactic theories need to solve. Nonetheless, the advantages of the mental models theory are even more obvious if other examples are considered. Another interesting case is that of the inferences such as the following:

\title{
David visited England.
}

Does it follow that David visited Paris or he visited England?" (Orenes \& Johnson-Laird, 2012, p. 375).

According to Orenes and Johnson-Laird (2012), most of the individuals do not respond positively in cases such as this (and this is something that they confirm experimentally) because one of the models of the conclusion is in contradiction with the premise (a description of the explanation of this phenomenon given by the mental models theory can also be found, e.g., in López-Astorga, 2015b, pp. 144ff). True, the Fully Explicit Models of the disjunction in the conclusion are these:
Paris
$\neg$ (Paris)
England
Paris

And the third one is incompatible with the premise (David did visit England), which explains why the participants in the study carried out by Orenes and Johnson-Laird tended to reject the conclusion of inferences such as this one.

But Orenes and Johnson-Laird (2012) further confirmed the predictions of the mental models theory proposing to their participants inferences such as the following as well:

\author{
"Lucia wore jewelry. \\ Therefore, Lucia wore the bracelet or she wore jewelry" (Orenes \& Johnson-Laird, 2012, p. 363; see also, e.g., López- \\ Astorga, 2015b, p. 145).
}

In these experimental conditions, the participants did accept the conclusion and, in Orenes and Johnson-Laird's view, the reason is not hard to understand either (this is accounted for, e.g., in López-Astorga, 2015b, p. 145, too). Modulation removes the controversial combination here, since the conclusion only has these two Fully Explicit Models: 
$\begin{array}{ll}\text { Bracelet } & \text { Jewelry } \\ \neg \text { (Bracelet) } & \text { Jewelry }\end{array}$

The third one, which would be inconsistent with the premise (it would provide that Lucia wore the bracelet and did not wear jewelry), is impossible. So, it is evident why the participants considered these inferences to be correct as well.

Following Orenes and Johnson-Laird's (2012) arguments, it can be said that this is also an important challenge for the syntactic or formal theories because, again, the last two inferences have identical formal structure:

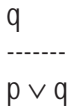

And in standard propositional calculus, as it is also well known, $[q] \vdash[p \vee q]$. It is hence very difficult to explain the results of these tasks from it or similar frameworks.

In my view, these facts prove that the mental models theory can be right that human reasoning can be about possibilities, and that the semantic content can be necessary to detect such possibilities. Nonetheless, I think that this does not mean that there is not a syntax in the human mind or that human reasoning has nothing to do with formal logic. From the models, whether Mental Models or Fully Explicit Models, formulae in the system K language can be built, and, thus, it is easy to come to the results expected by the mental models theory in that system. I show this below.

\section{Combinations of possibilities and $\mathrm{K}$}

As it is also well known, after Kripke and his work in modal logic (e.g., Kripke, 1959, 1962, 1963a, 1963b, 1965), a particular axiomatic system is called ' $\mathrm{K}$ ' in his honor. That system assumes standard logic and its operators (it is true that in $\mathrm{K}$ some of those operators are defined by means of others, but the final result is that all of them are a part of the system) and, in addition, includes two more operators: the one of necessity (' $\square$ ') and the one of possibility (' $\diamond$ '). Thus, these definitions hold:

$$
\begin{aligned}
& \square X==_{\text {df }} \neg \neg \neg X \\
& \diamond X==_{\text {df }} \neg \square \neg X
\end{aligned}
$$

The system also has a special rule and an additional axiom, but, as far as the aims of this paper are concerned, it is only necessary to consider the rule, which is usually named 'the necessitation rule':

$[N R]$ If $X$ is a theorem, then so is $\square X$.

But the point is, as said, that this system is clearly compatible with the derivations of conclusions made by the mental logic theory from its combinations of possibilities. However, to note that, another aspect needs to be explained: the way such possibilities can be transformed into well formed formulae in the $\mathrm{K}$ language. A first step in this direction can be to consider each model or possibility to be a conjunction and, therefore, each element of each model to be a conjunct. Really, this idea is not new, as it appears to be explicitly present in some previous works about the relationships between the possibilities of the mental models theory and logical forms (e.g., López-Astorga, 2014, 2015b, 2015c). Thus, for example, the Mental Model of the conditional could be expressed as follows:

$p \wedge q$

And its Fully Explicit Models could be transformed into these formulae:

$$
\begin{aligned}
& p \wedge q \\
& \neg p \wedge q \\
& \neg p \wedge \neg q
\end{aligned}
$$


Nevertheless, one more step would be necessary too. Given that the models represent possibilities, this characteristic of them should be also indicated. Evidently, $\mathrm{K}$ has the resources to do that. It is enough to resort to the operator $\diamond$ and complete the formulae with it. So, for instance, the truly suitable way to express the Fully Explicit Models of the conditional would be this one:

$$
\begin{aligned}
& \diamond(p \wedge q) \\
& \diamond(\neg p \wedge q) \\
& \diamond(\neg p \wedge \neg q)
\end{aligned}
$$

Now, it can be clearly noted that the models do not refer to facts, but only to possibilities, and, as shown in the next section, this formal expression of them is an important element to connect the mental models theory with $\mathrm{K}$. This point is developed below with the help of the same examples commented on above.

\title{
5. The predictions of the mental models theory and $\mathrm{K}$
}

The first example is that of the triangle and the circle. Given that its first premise is a conditional ('If there is a triangle then there is not a circle'), there are two options: only the Mental Model of that premise is detected and all of its Fully Explicit Models are identified. However, the problem is that, in both cases, that premise only refers, as explained, to possibilities (one when the Mental Model is detected and three when the Fully Explicit Models are so). In this way, individuals only know for sure what is indicated by the second premise, that is, that 'there is a circle'. Therefore, it seems to be justified to work in $\mathrm{K}$ just from this last datum in order to discover the consequences that can be derived from it, and whether or not such consequences are compatible with one or more of the models corresponding to the first conditional premise. Thus, it can be said that, in the case in which only the Mental Model of this last premise is identified, the formula that can be linked to that model is, as accounted for, $[\diamond(p \wedge \neg q)]$ (where ' $p$ ' refers to the triangle and ' $q$ ' represents the circle), and that the following deduction in $\mathrm{K}$ shows in a obvious way why nothing is inferred:

$$
\begin{aligned}
& {[1] q \rightarrow q} \\
& \text { [2] } q \rightarrow \neg p \vee q \\
& {[3] q \rightarrow \square(\neg p \vee q)} \\
& \text { [4] } q \rightarrow \neg \vee \neg(\neg p \vee q) \\
& {[5] q \rightarrow \neg \vee(p \wedge \neg q)}
\end{aligned}
$$

(from standard calculus)

(from 1)

(from 2 by NR)

(from 3 by the definition of $\square$ )

(from 4)

Indeed, given [q], i.e., the fact that 'there is a circle', the model must be rejected, since, as shown in step [5], in a scenario in which $[q]$ is true, $[p \wedge \neg q]$ cannot be possible. Accordingly, to make an inference here, it is necessary, as also explained, to detect all of the Fully Explicit Models.

When that happens, the formulae representing the possibilities are $[\diamond(p \wedge \neg q)],[\diamond(\neg p \wedge \neg q)]$, and $[\diamond(\neg p \wedge q)]$. But again steps [1] to [5] reveal that the first formula must be rejected. Likewise, something similar occurs with the second formula, as it can be seen in the next steps:

$$
\begin{aligned}
& {[6] q \rightarrow p \vee q} \\
& {[7] q \rightarrow \square(p \vee q)} \\
& {[8] q \rightarrow \neg \diamond \neg(p \vee q)} \\
& {[9] q \rightarrow \neg \vee(\neg p \wedge \neg q)}
\end{aligned}
$$

\author{
(from 1) \\ (from 6 by NR) \\ (from 7 by the definition of $\square$ ) \\ (from 8)
}

Clearly, step [9] indicates that [q] is incompatible with $[\diamond(\neg p \wedge \neg q)]$. The only possibility that remains is hence that of the third formula, which cannot be demonstrated to be false if [q] is true in K. For this reason, it can be thought that $[\diamond(\neg p \wedge q)]$ is the formula that describes the only scenario that can be real and that, therefore, [ $\neg p]$ must be true.

Nonetheless, the situation changes in the example of the musical instrument and the flute. Now, ' $p$ ' stands for the musical instrument and ' $q$ ' denotes the flute, and, although the account in the case in which only the Mental Model of the first conditional premise is detected can be exactly the same as the previous one, the circumstance is very different when its Fully Explicit Models are detected. As indicated, the first premise of this example has only two Fully Explicit Models, $[\diamond(p \wedge \neg q)]$ and $[\diamond(\neg p \wedge \neg q)]$, and, as shown in steps [1] to [5] and steps [1] and [6] to [9] respectively, both of them are 
incompatible with a situation in which [q] is the case. So, it makes sense that people do not tend to conclude that she did not play a musical instrument given the premises, since that would only be so in a scenario that cannot be considered because it is not really possible (the scenario in which she plays a flute and does not play a musical instrument).

In connection with the example of David, England and Paris, it can be claimed that what changes now is the perspective. Given that the sentence that refers to different possibilities is now the conclusion, and not a premise, it seems that what must be checked is whether or not all of such possibilities are consistent with the premise, and not, as in the previous cases, which the model (or models) describing the real situation is (or are). If we assume that the disjunction is inclusive, that ' $q$ ' indicates that David visited England, and that ' $p$ ' refers to the fact that David visited Paris, based on all it has been said above, those possibilities would be the following:

$$
\begin{aligned}
& \diamond(p \wedge q) \\
& \diamond(\neg p \wedge q) \\
& \diamond(p \wedge \neg q)
\end{aligned}
$$

The two first formulae are not a problem because $\mathrm{K}$ cannot prove that they are false if the premise [q] is true. This, in addition, also removes the problem of the interpretation of the disjunction (as inclusive or exclusive), since, if it were exclusive, the only difference would be that the first formula, $[\diamond(p \wedge q)]$, would not be considered. However, given that this last formula, as mentioned, is not incompatible with [q], it is a difficulty neither it is included in the models set nor it is not. The real problem is the third formula, and that can be noted in step [5], which reveals that, when [q] is the case, $[\diamond(p \wedge$ $\neg q)$ ] cannot be so. Accordingly, the deductions of the system fit the predictions of the mental models theory in this example as well, since such deductions are not compatible with all of the formulae (possibilities) related to the conclusion, and hence also show why people tend to reject the conclusion in this kind of task.

Furthermore, after this explanation, what happens in the example of Lucia, the bracelet, and jewelry, if it is assumed that ' $q$ ' stands for the situation in which Lucia wears jewelry and ' $p$ ' denotes the scenario in which she wears the bracelet, is evident. The third formula, $[\diamond(p \wedge \neg q)]$, cannot be taken into account because it is not possible that somebody wears a bracelet and does not wear jewelry. Therefore, there is no formula to consider that is incompatible with [q] in $\mathrm{K}$ and the conclusion can hence be accepted.

\section{Conclusions}

Accordingly, it appears that it is still possible to support the idea that there is a syntax of thought. That syntax do not need to be similar to the one proposed by the mental logic theory. It can be a deeper syntax that works at models level, and not directly at natural language level, as the mental logic theory seems to claim. In this way, the most important point of this paper is that, even accepting the general theses of the mental models theory, it continues to make sense to speak about mental formal processes and a syntax of any kind.

Of course, it is very probable that the exact mental process made by individuals does not match a strict application of the formal rules and requirements of a system such as K. However, the fact that this last system can lead to conclusions absolutely compatible with those of the mental models theory means that we cannot totally ignore the possibility that human reasoning is, at least to some extent, linked to logic yet.

A reasonable option in this regard can be, following the basic ideas of papers such as, e.g., those of LópezAstorga $(2014,2015 b, 2015 c)$, to suppose two phases in the human reasoning activity. A first phase would be that in which the expressions in natural language are interpreted. This process would be always semantic and based on possibilities and the content of the sentences. Undoubtedly, this would be a crucial phase and it appears to be obvious that the discovery of this type of processes is a great finding of the mental models theory.

Nonetheless, this does not mean that a subsequent syntactic or formal process is not possible. That process would be the phase in which the inferences are actually made from the combinations of possibilities detected in the first phase. The consistency found in this paper between the predictions of the mental models theory and what can be inferred in a system such as $\mathrm{K}$ shows that this idea is not absurd. Clearly, it needs to be supported by further research, as, for example, it is necessary to check whether or not $\mathrm{K}$ is also compatible with other results reported in the literature that the mental models theory can explain, but, as said, it is not a baseless idea.

Furthermore, as it is well known, there are several problems that all of the syntactic reasoning theories and any intent to describe the syntax of thought, including the proposal of this paper, have to face. One of them is, for instance, the fact that standard calculus (and $\mathrm{K}$ is based on standard calculus) enables infinite conclusions. For example, $[\mathrm{p} \wedge \mathrm{p}]$ 
can be inferred from [p], $[p \wedge p \wedge p]$ can be inferred from $[p \wedge p],[p \wedge p \wedge p \wedge p]$ can be inferred from $[p \wedge p \wedge p]$, and so on (see, e.g., Johnson-laird et al., 2015, p. 201). On the other hand, another difficulty is, for example, that related to the principle of explosion (Ex Falso Quodlibet Sequitur), which allows doing something that people do not usually do: to derive any conclusion from a contradiction (see, e.g., Johnson-Laird, 2010, p. 204).

It is absolutely true that the syntactic frameworks such as that of the mental logic theory have given solutions to these and other problems. Thus, this last theory removes the difficulty of the infinite conclusions by stating that not all of the formal schemata in the human mind are of the same type, and that some of them (called 'Feeder Schemata') are only applied when useful (see, e.g., Braine \& O'Brien, 1998c, p. 83). Likewise, this same theory tries to overcome the inconveniences of the principle of explosion by affirming that, in the human mind, contradictions are not related to that principle, but only to the 'Reductio ad Absurdum strategy' (see, e.g., Braine \& O'Brien, 1998c, p. 83; Braine \& O'Brien, 1998d, p. 206). In fact, the theory proposes even a program accounting for the order in which people generally use the different schemata (see, e.g., Braine \& O'Brien, 1998c, pp. 82-83, Table 6.2). However, this theory seems to continue to have another important problem of the formal theories: they need to explain the exact process why logical forms are recovered from the expressions in natural language (see, e.g., Johnson-Laird et al., 2015, p. 202). As said, the mental logic theory seems to apply its schemata to sentences in natural language and, as it is known, the same words in natural language do not necessarily lead to the same logical forms. This is a difficulty clearly solved by the mental models theory and, for reasons such as this one, it appears to be justified to think about a syntax of thought that do not ignore the potential of the mental models theory. This paper has been an attempt in this way and, although there is no doubt that further study is necessary to remove some of its limitations, perhaps it has revealed a possible direction that the research on the relationships between syntax and semantics in the human mind should follow.

\section{References}

Braine, M. D. S. \& O'Brien, D. P. (Eds.) (1998a). Mental Logic. Mahwah, NJ: Lawrence Erlbaum Associates, Inc., Publishers.

Braine, M. D. S. \& O'Brien, D. P. (1998b). How to investigate mental logic and the syntax of thought. In M. D. S. Braine \& D. P. O'Brien (Eds.), Mental Logic (pp. 45-61). Mahwah, NJ: Lawrence Erlbaum Associates, Inc., Publishers.

Braine, M. D. S. \& O'Brien, D. P. (1998c). The theory of mental-propositional logic: Description and illustration. In M. D. S. Braine \& D. P. O'Brien (Eds.), Mental Logic (pp. 79-89). Mahwah, NJ: Lawrence Erlbaum Associates, Inc., Publishers.

Braine, M. D. S. \& O'Brien, D. P. (1998d). A theory of if: A lexical entry, reasoning program, and pragmatic principles. In M. D. S. Braine \& D. P. O'Brien (Eds.), Mental Logic (pp. 199-244). Mahwah, NJ: Lawrence Erlbaum Associates, Inc., Publishers.

Byrne, R. M. J. \& Johnson-Laird, P. N. (2009). "If" and the problems of conditional reasoning. Trends in Cognitive Science, 13(7), 282287.

Fodor, J. (1975). The Language of Thought. Cambridge, MA: Harvard University Press.

Gentzen, G. (1935). Untersuchungen über das logische Schließen I. Mathematische Zeitschrift, 39, 176-210.

Johnson-Laird, P. N. (2006). How We Reason. Oxford, UK: Oxford University Press.

Johnson-Laird, P. N. (2010). Against logical form. Psychologica Belgica, 5(3/4), 193-221.

Johnson-Laird, P. N. (2012). Inference with mental models. In K. J. Holyoak \& R. G. Morrison (Eds.), The Oxford Handbook of Thinking and Reasoning (pp. 134-145). New York, NY: Oxford University Press.

Johnson-Laird, P. N. (2015). How to improve thinking. In R. Wegerif, L. Li, \& J. C. Kaufman (Eds.), The Routledge International Handbook of Research on Teaching Thinking (pp. 80-91). Abingdon, UK, \& New York, NY: Routledge.

Johnson-Laird, P. N., Khemlani, S., \& Goodwin, G. P. (2015). Logic, probability, and human reasoning. Trends in Cognitive Sciences, 19(4), 201-214.

Khemlani, S., Orenes, I., \& Johnson-Laird, P. N. (2012). Negation: A theory of its meaning, representation, and inference. Psychological Review, 109(4), 646-678.

Khemlani, S., Orenes, I., \& Johnson-Laird, P. N. (2014). The negation of conjunctions, conditionals, and disjunctions. Acta Psychologica, 151, 1-7.

Kripke, S. A. (1959). A completeness theorem in modal logic. Journal of Symbolic logic, 24(1), 1-14.

Kripke, S. A. (1962). The undecidability of monadic modal quantification theory. Zeitschrift für Mathematische Logik und Grundlagen der Mathematik, 8, 113-116.

Kripke, S. A. (1963a). Semantical considerations in modal logic. Acta Philosophica Fennica, 16, 83-94.

Kripke, S. A. (1963b). Semantical analysis of modal logic I: Normal modal propositional calculi. Zeitschrift für Mathematische Logik und Grundlagen der Mathematik, 9, 67-96.

Kripke, S. A. (1965). Semantical analysis of modal logic II: Non-normal modal propositional calculi. In J. W. Addison, L. Henkin, \& A. Tarski (Eds.), The Theory of Models: Proceedings of the 1963 International Symposium at Berkeley (pp. 206-220). Amsterdam, The Netherlands: North-Holland Publishing Co.

López-Astorga, M. (2014). Mental models and syntactic rules: A study of the relations between semantics and syntax in inferential processes. Analele Universitatii din Craiova, Seria Filosofie, 33(1), 107-117.

López-Astorga, M. (2015a). Diodorus Cronus and Philo of Megara: Two accounts of the conditional. Rupkatha, 7(3), 9-16. 
López-Astorga, M. (2015b). The disjunction introduction rule: Syntactic and semantic considerations. Pragmalingüistica, 23, 141-149.

López-Astorga, M. (2015c). Evolved mechanisms versus underlying conditional relations. Studies in Logic, Grammar and Rhetoric, 40(53), 241-253.

López-Astorga, M. (2016a). Towards an updated reasoning formal theory. Aufklärung, 3(1), 11-32.

López-Astorga, M. (2016b). The first rule of Stoic logic and its relationship with the indemonstrables. Tópicos. Revista de Filosofia, 50, 923.

López-Astorga, M. (2016c). Does Aristotelian logic describe human reasoning? Valid syllogisms and canonical models. Khazar Journal of Humanities and Social Sciences, 19(2), 5-27.

Macnamara, J. (1986). A Border Dispute: The Place of Logic in Psychology. Cambridge, MA: Massachusetts Institute of Technology (MIT) Press.

Oakhill, J. \& Garnham, A. (Eds.) (1996). Mental Models in Cognitive Science. Essays in Honour of Phil Johnson-Laird. Hove, UK: Psychology Press.

O'Brien, D. P. (2009). Human reasoning includes a mental logic. Behavioral and Brain Sciences, 32, 96-97.

O'Brien, D. P. (2014). Conditionals and disjunctions in mental-logic theory: A response to Liu and Chou (2012) and to López-Astorga (2013). Universum, 29(2), 221-235.

O'Brien, D. P. \& Li, S. (2013). Mental logic theory: A paradigmatic case of empirical research on the language of thought and inferential role semantics. Journal of Foreign Languages, 36(6), 27-41.

Orenes, I. \& Johnson-Laird, P. N. (2012). Logic, models, and paradoxical inferences. Mind \& Language, 27(4), 357-377.

Ragni, M., Sonntag, T., \& Johnson-Laird, P. N. (2016). Spatial conditionals and illusory inferences. Journal of Cognitive Psychology, 28(3), 348-365. 